\title{
PARIS : un logiciel de simulation d'états radioécologiques post-accidentels
}

\author{
Ph. RENAUD*, H. MAUBERT*, D. ROBEAU* \\ I. LINGE**, O. PAVLOVSKY**, I. OSSIPIANTS**
}

(Manuscrit reçu le 30 octobre 1996, révisé le 9 mai 1997, accepté le 21 juin 1997)

RÉSUMÉ

ABSTRACT
Le logiciel PARIS (Post-Accidental Radiological Imitation System) a pour fonction de générer des situations post-accidentelles, en simulant les différents flux de données, notamment des mesures, tels qu'ils pourraient parvenir aux experts dans un cas réel. Les situations proposées sont particulièrement réalistes car établies par analogie avec celles observées après l'accident de Tchernobyl. Plusieurs fonctions témoignent de ce réalisme : la prise en compte de la capacité de mesure, des mouvements de population, des dispositions de protection prises par les autorités... Les bases de données associées sont d'une grande richesse qui rend compte de la diversité des informations que les experts auraient à traiter en cas de crise. Le logiciel PARIS constitue ainsi une synthèse informatisée et fonctionnelle des enseignements opérationnels que l'on peut tirer de l'accident de Tchernobyl. En ce sens il présente un grand intérêt pour la préparation des experts à la gestion des situations post-accidentelles. Lors d'exercices de simulation de crise, les données générées par PARIS peuvent être considérées comme une réalité virtuelle à laquelle sont confrontés les experts disposant des résultats prévisionnels d'outils de calculs et d'aide à la décision comme ASTRAL.

Paris, a software for simulation of post-accidental radioecological situations. The PARIS software (Post-Accidental Radiological Imitation System) has for function to generate post-accidental situation, by simulating the different data flows, notably measurements results, such as they could reach the experts in a real-life situation. Situations proposed are particularly realistic because established by analogy with situations observed after the Chernobyl accident. Many functions reveal this realism: the taking into account of measurements capacity, population movements, the consideration of countermeasures capacity of application and their real efficiency... The data bases used are very extensive which allows diversity of information that the experts will have to process in such a situation. The PARIS software constitutes a computerized functional synthesize of operational lessons that one can draw from the Chernobyl accident. In this sense, it presents a considerable interest for preparing experts in the management of post-accidental situations. During crisis exercices, data generated by PARIS can be considered as a virtual reality. Experts can compare results from calculation tools like ASTRAL, with this virtual reality.

\footnotetext{
* Institut de Protection et de Sâreté Nucléaire (IPSN).

** Institut Russe de Sûreté Nucléaire (IBRAE).
} 


\section{Introduction}

PARIS (post-accidental radiological imitation system) est un logiciel développé par l'institut russe de sûreté nucléaire IBRAE, à la demande de l'Institut de Protection et de Sûreté Nucléaire (IPSN).

Il a pour fonction de générer des situations post-accidentelles, en simulant les différents flux de données, notamment des résultats de mesures, tels qu'ils pourraient parvenir aux experts dans un cas réel.

Basé sur des mesures effectuées suite à l'accident de Tchernobyl dans les régions de Kaluga et Briansk en Russie, et de Gomel en Biélorussie, les deux premières versions du logiciel PARIS ne sont utilisables que dans les districts (départements) correspondants à ces trois «Oblasts». Les corrélations utilisées pour modéliser les résultats de mesures ne permettent pas actuellement l'adaptation à d'autres régions; de même que le choix d'une date de dépôt autre que celle fixée au $1^{\text {er }}$ mai 1990.

L'objectif de ce document est de présenter le logiciel PARIS, son principe de fonctionnement, sa structure, et de souligner les éléments qui témoignent du réalisme des simulations générées. Différents écrans du logiciel sont ainsi présentés et commentés afin d'illustrer certaines fonctions. Ils apparaissent en langue anglaise tels que dans l'interface utilisateur.

L'utilisation qui est faite de ce logiciel lors des exercices de gestion de situations post-accidentelles est également présentée, en soulignant notamment la complémentarité entre PARIS et le système ASTRAL de calculs et d'aide à la décision.

\section{Présentation du logiciel PARIS}

\subsection{Principe de fonctionnement}

Le principe de fonctionnement de PARIS est schématisé dans la figure 1. À partir d'un terme source, qui caractérise les rejets de radioactivité et en prenant en compte différentes données relatives aux zones touchées, le logiciel PARIS fournit des résultats de mesures. Ces valeurs sont ensuite utilisées pour des estimations de doses et, en fonction de différents critères d'intervention, vont conduire suivant le cas à l'application de contre-mesures. Certaines de ces contre-mesures auront des répercussions sur les zones concernées, sur les nouveaux résultats de mesures, et sur les nouvelles estimations de doses. 


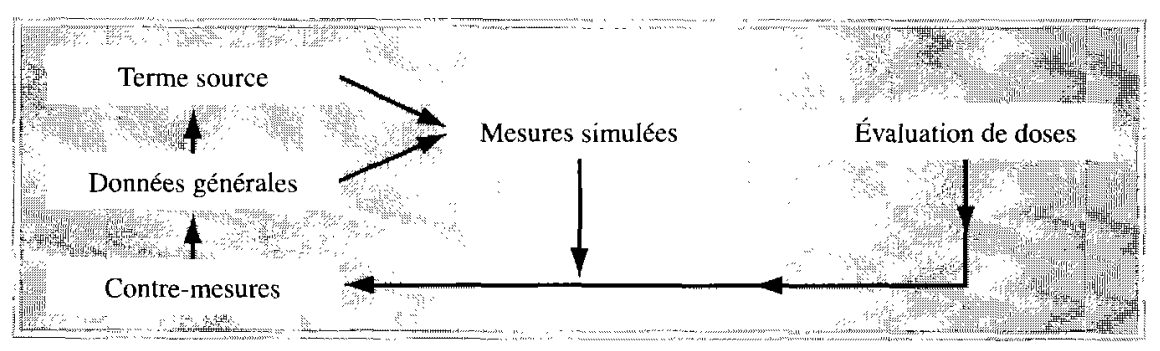

Fig. 1. - Schéma de principe du logiciel PARIS.

Skeleton diagram of PARIS software.

Ainsi dans PARIS la modélisation n'est pas utilisée pour l'étude prévisionnelle d'impact, mais pour simuler l'obtention de résultats de mesures, sur la base de corrélations de mesures réelles effectuées après l'accident de Tchernobyl. Cette originalité se traduit par une structure particulière, axée sur l'utilisation et la génération de bases de données.

\subsection{Architecture}

La figure 2 présente les trois ensembles de bases de données et les deux éléments fonctionnels qui les relient. Les quatre bases de données initiales contiennent respectivement :

- les informations relatives aux 60 radionucléides considérés dans PARIS : symboles, périodes radioactives, valeurs à défaut du terme source, facteurs de doses...

- les informations administratives et socio-démographiques des zones susceptibles d'être concernées par les rejets,

- les données agronomiques relatives à ces mêmes zones ainsi que les rations alimentaires humaines et animales,

- les éléments relatifs à la caractérisation des contre-mesures.

L'utilisateur peut caractériser les rejets en définissant le terme source : activités présentes dans le cour et fractions émises (création de la Base [Terme source]), sélectionner et éventuellement modifier les données géographiques (Bases [Données géographiques] et [D. agricoles]), et déterminer les contremesures qu'il souhaite appliquer (Base [Contre-mesures]) (cf. $\S 2.3 .1$ ). A ces données spécifiques à l'étude viennent s'ajouter celles relatives au scénario post-accidentel (nature et nombre de résultats de mesures, définition de phases d'interventions...). 
Neuf bases de résultats de mesures sont alors générées :

- [Débits de dose], [Sols] et [Eaux] fournissent respectivement : des mesures de débits de doses bg $\left(\mu \mathrm{Sv} \mathrm{h} \mathrm{h}^{-1}\right)$, de densités de contamination du sol (dépôts en $\left.\mathrm{kBq} \mathrm{m}^{-2}\right)$ et de contaminations des eaux $\left(\mathrm{Bq} \mathrm{l}^{-1}\right)$, pour chaque radionucléide et en différents lieux,

- [Prod. fermiers], [Prod. agricoles] et [Denrées aliment.] fournissent les concentrations de différents produits agricoles végétaux et animaux, ainsi que des principales denrées alimentaires dérivées; et ceci pour chaque radionucléide et pour des échantillons prélevés en différents lieux,

- [Dosim. indiv], [Compt. thyroïde] et [Compt. corporel] fournissent des dosimètres individuelles $(\mathrm{mSv})$, des comptages thyroïdiens $(\mathrm{Bq})$ et des comptages corporels globaux $(\mathrm{Bq})$ de différents individus dont le lieu de domicile, le sexe, l'âge et la catégorie socio-professionnelle sont identifiés.

Pour chaque mesure, la date et le lieu du prélèvement de l'échantillon sont donnés. Les effets des éventuelles contre-mesures sont par ailleurs pris en compte.

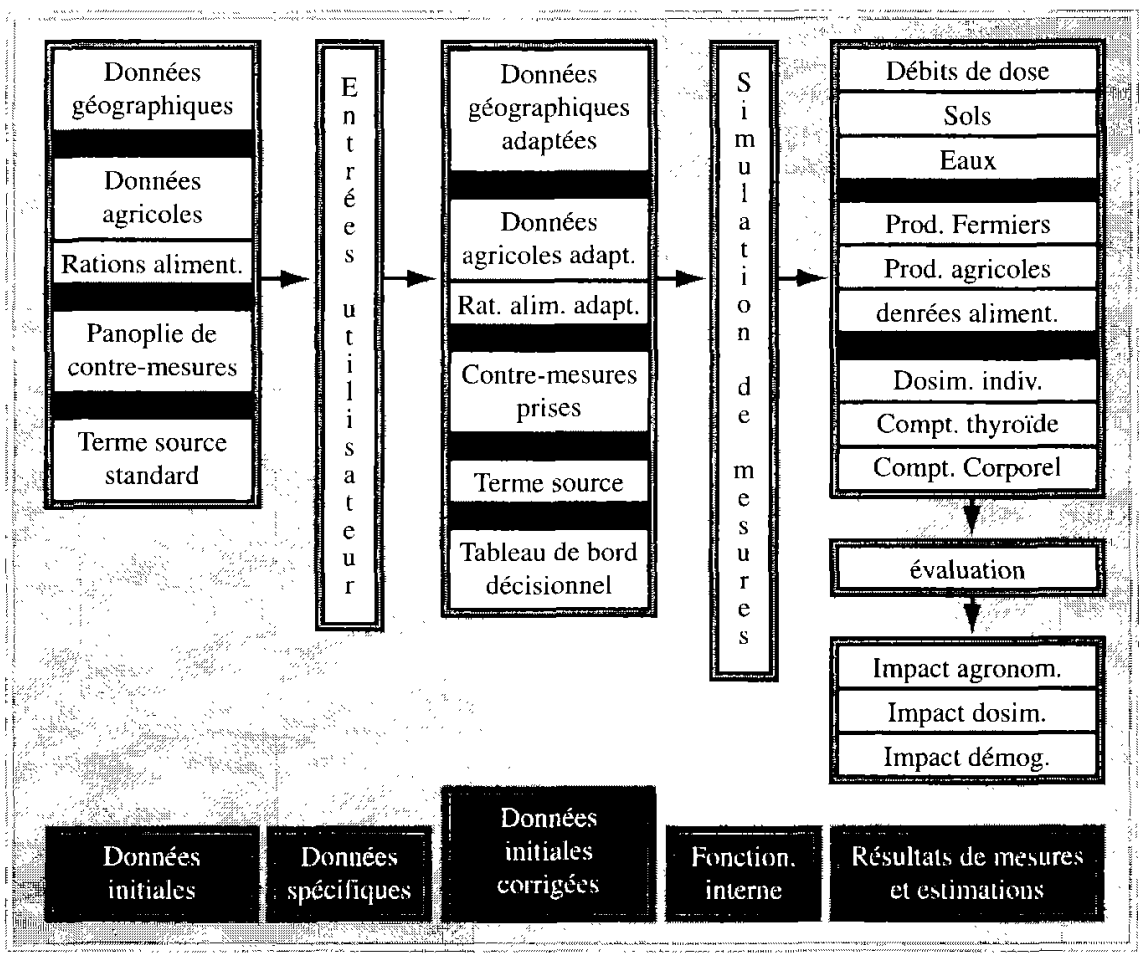

Fig. 2. - Bases de données de PARIS.

PARIS Data base. 
À partir de ces données, différentes évaluations sont faites :

- La base [Impact agronomique] contient les estimations des surfaces concernées par différents niveaux de densité de contamination. Il est par exemple possible de connaître dans un espace agricole la surface contaminée par un dépôt de césium compris entre 37 et $185 \mathrm{kBq} \mathrm{m}^{-2}$.

- La base [Impact démographique)] est une nouvelle version de la base [Données géographiques] qui prend en compte les mouvements de population résultant des évacuations et relogements (les dates de début et de fin d'évacuation sont précisées).

- La base [Impact dosimétrique] contient des estimations de doses (mSv) : efficace, thyroïde, poumon, moelle osseuse... avec les contributions des voies d'atteintes: immersion dans le panache, ingestion, exposition externe, inhalation...

\subsection{Fonctionnalités particulières}

Parmi les nombreuses fonctionnalités de PARIS, deux témoignent plus particulièrement du souci de réalisme qui caractérise ce logiciel. II s'agit de la prise en compte des contre-mesures et de l'utilisation du nombre de mesures.

\subsubsection{Prise en compte des contre-mesures}

La figure 3 présente l'unique écran de l'interface utilisateur et la fenêtre correspondant au menu «choix des contre-mesures».

Après avoir défini le terme source par les menus « facteur d'échelle » et « composition isotopique », ainsi que la date d'étude (A sur la Fig. 3), l'utilisateur peut définir jusqu'à cinq «dates de prise de décisions » (B sur la Fig. 3) entre la date de dépôt et la date d'étude. Ces dates délimitent des phases post-accidentelles durant lesquelles différents jeux de contre-mesures peuvent être appliqués. Il est ainsi possible de choisir dans une liste de 9 contre-mesures (C1 à $\mathrm{C} 9$ ) celles que l'on souhaite appliquer sur chaque phase. Suivant le cas certaines ne peuvent pas être appliquées. C'est le cas par exemple du traitement préventif à l'iode réservé à une phase antérieure au dépôt.

Les contre-mesures choisies doivent être alors caractérisées sur chaque phase. Il s'agit de définir le critère ou niveau d'intervention, l'efficacité intrinsèque de la contre-mesure, mais également de déterminer les capacités d'application, et son degré d'application. Le critère d'intervention peut être exprimé en terme de dose, de densité de contamination ou de concentration suivant le cas. Le degré d'application correspond au fait que la contre-mesure, pour de multiples raisons, peut ne pas avoir été appliquée partout où elle était requise. L'efficacité d'application d'une contre-mesure sur une zone ou une population est la résultante de son efficacité intrinsèque et de son degré d'application. 


\begin{tabular}{|c|c|c|c|c|c|c|c|c|c|}
\hline 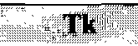 & $\mathrm{Cl}$ & C2 & c3 & C4 & C5 & c6 & C7 & cg & $\mathrm{Cg}$ \\
\hline 30.04 .90 & - & + & + & - & - & - & - & - & - \\
\hline 10.05 .90 & + & & & & + & & & & \\
\hline 25.05 .90 & + & & & + & & & & & \\
\hline 01.03 .91 & & & & & + & + & + & + & + \\
\hline 04.07.92 & & & & & & + & & & + \\
\hline
\end{tabular}

\begin{tabular}{|c|}
\hline INPUT OF THE DATA \\
\hline Scaling factor \\
Isotop composition \\
Time after contamination (A) \\
Dates of making decisions (B) \\
Choice of counterneasures \\
Countermeasure performances \\
List of district \\
List of data bases \\
\hline
\end{tabular}

Cl Evaluation/Resttlement

C2 Iodine Preventive Treatement for children

C3 Iodine Preventive Treatement for adults

C4 Decontamination

C5 Removal of children

C6 Limitation of food consumption

C7 Mineral fertilizers placement

C8 Decp plowing

C9 Application of ferrocyanides

Fig. 3. - Fenêtre de l'Interface Homme Machine (IHM) permettant le choix des contre-mesures Window of the User Interface allowing choice of countermeasures.

La figure 4 présente le cas de l'évacuation. Pour la phase qui débute le 10 mai 1990 et qui dure jusqu'au 25 mai, le critère d'évacuation est de $500 \mathrm{mSv} / \mathrm{an}$. Durant cette phase, la capacité d'évacuation est de 1000 personnes par jour. On s'attend à ce que l'on ne puisse évacuer que $10 \%$ des personnes qui devraient l'être. Pour la phase suivante ces critères peuvent évoluer. Le niveau d'intervention peut par exemple être inférieur et la capacité d'évacuation peut augmenter.

Les éléments relatifs aux autres contre-mesures sont présentés dans le tableau $\mathrm{I}$.

La prise en compte de la capacité d'application et de l'efficacité réelle introduit une touche de réalisme sur deux aspects souvent négligés par les experts : la faisabilité et le gain réel de l'application des contre-mesures. 


\begin{tabular}{|c|c|c|c|c|c|c|c|c|c|}
\hline TK & C1 & (q4) & $\mathrm{c}$ & C4 & $\overline{C 5}$ & C6 & c7 & Cs: & c9 \\
\hline 30.04 .90 & - & + & + & - & - & - & - & ${ }^{\circ}$ & • \\
\hline 10.05 .90 & + & & & & + & & & & \\
\hline 25.05 .90 & + & & & + & & & & & \\
\hline 01.03.91 & & & & & + & + & + & + & + \\
\hline 04.07 .92 & & & & & & + & & & + \\
\hline
\end{tabular}

\begin{tabular}{|c|}
\hline INPUT OF THE IDATA \\
\hline Scaling factor \\
\hline Isotop composition \\
\hline Time after contamination \\
\hline Dates of making decisions \\
\hline 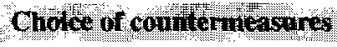 \\
\hline List of district \\
\hline List of data bases \\
\hline
\end{tabular}

Fig. 4. - Définition des caractéristiques de l'évacuation.

Specification of evacuation characteristics.

\subsubsection{Utilisation de la capacité de mesure}

L'utilisateur définit pour les différents types de mesures, le nombre de résultats de mesures que le logiciel va lui fournir. Ces résultats vont se répartir dans le temps et l'espace, en fonction de critères prédéfinis.

Le nombre de mesures sur un site est relié à l'importance du site (nombre d'habitants) mais également au niveau de contamination attendu (densité de contamination théorique DTC). Le tableau II présente le nombre de mesures d'échantillons de sol attribué par PARIS à 7 sites du district de Ljudinovo, pour un nombre total de 500 mesures de ce type défini par l'utilisateur sur ce district. La petite commune de Grjada qui ne compte que 89 habitants est presque autant étudiée que Manino (627 habitants) qui est significativement moins contaminée. Les grandes villes comme Ljudinovo font dans tous les cas l'objet d'un grand nombre de mesures.

Le nombre total de mesures défini par l'utilisateur est également réparti dans le temps avec des dynamiques variables. Les mesures de débits de doses seront les plus nombreuses dans les jours qui suivent les dépôts. Les mesures de sols, moins nombreuses, seront réparties uniformément dans le temps, alors que les comptages thyroïdes et surtout anthropo-gammamétriques interviendront plus tard. À chaque résultat de mesure est attaché un lieu et une date. 
Des estimations sont ensuite faites à partir des mesures : densité de contamination moyenne, maximum, minimum, dose intégrée, doses prévues, etc. La précision et la fiabilité de ces estimations augmentent le plus souvent avec le nombre de mesures. Par exemple, le tableau III présente des estimations de la densité de contamination en césium 137 en relation avec le nombre de mesures (entre parenthèses). Ces estimations sont à comparer avec la valeur réelle (théorique) de la densité de contamination (DTC). Cependant, le hasard peut faire que, comme dans la réalité, une mesure unique permette une meilleur appréciation que 8 mesures. C'est le cas pour Kotovichi.

Ces valeurs sont comparées aux critères d'intervention (cf. Paragr. 2.3.1) et peuvent conduire à l'application de contre-mesures. De ce fait, comme dans la réalité, la décision d'appliquer des contre-mesures repose sur des estimations dont la fiabilité dépend également du nombre de mesures. Le tableau IV montre que si le nombre de mesures est insuffisant, il en résulte une sous-estimation ou une surestimation des doses qui peut conduire à ne pas évacuer des communes qui devraient l'être (cas de Grjada) ou l'inverse (cas de Kotovichi).

TABLEAU I

Caractérisation des contre-mesures.

Characterization of countermeasures.

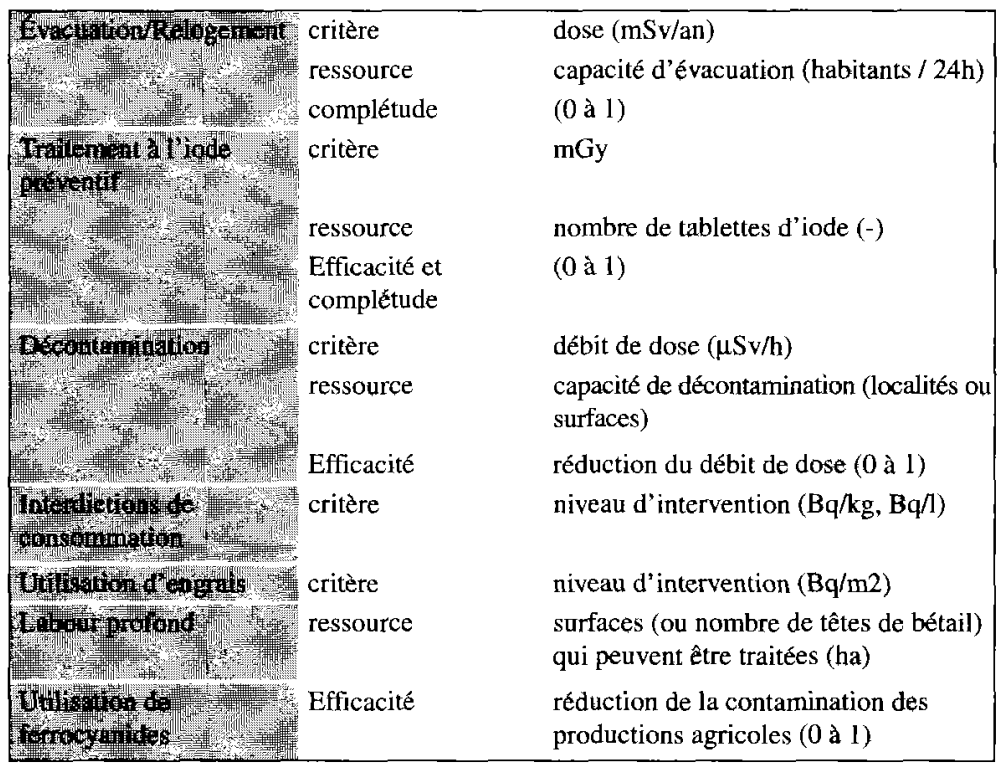




\section{TABLEAU II}

Nombre de mesures par un site en relation avec sa population et sa contamination.

Number of measures attributed to a site in relation to its population and density of contamination.

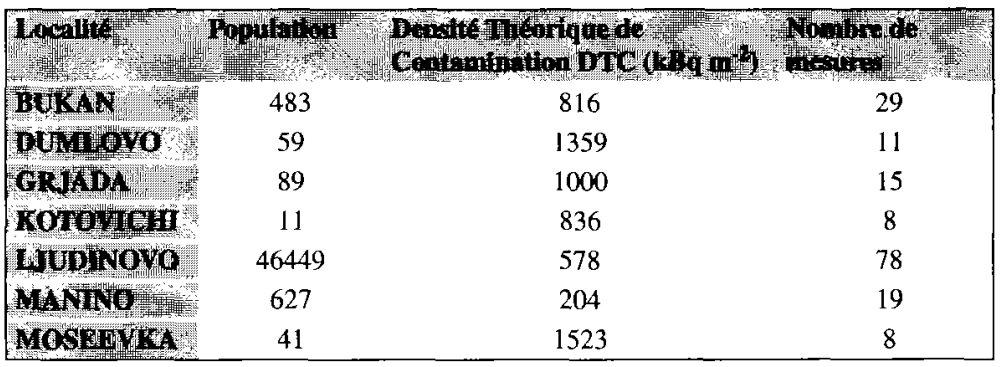

TABLEAU III

Estimations de la contamination en $137 \mathrm{Cs}$ en relation avec le nombre de mesures de sol.

Estimates of density of contamination in $137 \mathrm{Cs}$ in relation to number of measurements of soil samples.

\begin{tabular}{|c|c|c|c|c|}
\hline 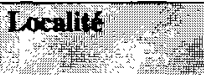 & Min & \multicolumn{3}{|c|}{ 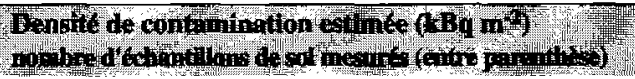 } \\
\hline BUAN & 816 & $583(1)$ & $752(15)$ & $773(29)$ \\
\hline DUNMO IO & 1359 & $(0)$ & $1309(4)$ & $1354(11)$ \\
\hline 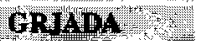 & 1000 & $1371(1)$ & $1027(13)$ & $1030(15)$ \\
\hline WOMMOHW & 836 & $826(1)$ & $706(8)$ & $714(8)$ \\
\hline 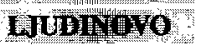 & 578 & $547(2)$ & $559(18)$ & $580(78)$ \\
\hline NHAn & 204 & $254(1)$ & $214(4)$ & $193(19)$ \\
\hline Mongror. & 1523 & $(0)$ & $1399(6)$ & $1421(8)$ \\
\hline
\end{tabular}

\section{TABLEAU IV}

Évacuation de localités en relation avec le nombre de mesures et donc la précision des estimations.

Evacuation of districts in relation to the number of measurements and therefore accuracy of estimations.

\begin{tabular}{|c|c|c|c|c|}
\hline Irenlet & 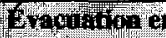 & bom tor an no & Hethanplino & 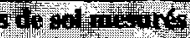 \\
\hline W & TI & athosphes & $\mathrm{SO}_{\mathrm{L}}$, sares & 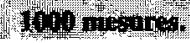 \\
\hline 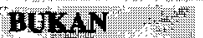 & Non & Non & Non & Non \\
\hline DUMUNO & Non & Oui & Out & Out \\
\hline 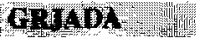 & Non & Non & Oui & Col \\
\hline WOAONA II & Non & Non & Qui & Non \\
\hline HIDUNOW & Non & Non & Non & Non \\
\hline MI & Non & Non & Non & Non \\
\hline 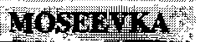 & Non & Out & Oui & Wou \\
\hline
\end{tabular}




\section{Exemples d'utilisations du logiciel PARIS}

\subsection{Gestion des mesures et exploitation des résultats}

Un des principaux problèmes pour l'expert est de caractériser les zones contaminées. Au-delà de la phase de calculs prévisionnels, cela repose sur l'interprétation de résultats de mesures étalées dans le temps et l'espace. Combien de mesures faut-il pour obtenir une image crédible de la situation?

PARIS fournit le nombre de mesures demandé; la génération aléatoire des données reproduit la variabilité de ce qui serait observé dans la nature. Il peut ainsi permettre aux experts de s'entraîner à optimiser les moyens de mesures (et de prélèvements) à mettre en œuvre pour asseoir un jugement fiable. 11 reste alors le problème du passage progressif des résultats de calculs aux résultats de mesures. Il s'agit d'un problème complexe, thème de prochains travaux de collaboration entre l'IPSN et L'IBRAE.

\subsection{Utilisation combinée d'ASTRAL et de PARIS lors d'exercices de gestion de situations post-accidentelles}

ASTRAL (Assistance Technique en Radioprotection post-Accidentelle) est un système développé par l'IPSN et destiné à l'assistance aux experts et autorités pour la caractérisation d'une situation post-accidentelle et la prise de décision (Maubert et al., 1996).

L'utilisation combinée de PARIS et d'ASTRAL présente un grand intérêt dans le cadre d'exercices de gestion de situations post-accidentelles. PARIS est utilisé pour simuler de manière réaliste des situations que des experts ont la charge de gérer avec l'assistance du logiciel ASTRAL. Les données issues de PARIS sont considérées comme une réalité virtuelle et ASTRAL peut, de par sa conception, s'adapter tout naturellement aux situations générées par PARIS.

Deux exercices internationaux, organisés principalement par la Russie et la France suivant ce principe ont déjà eu lieu : le premier en juin 1993 portait sur la région de Kaluga (IPSN-IBRAE, 1994), le second en mai 1995 avait pour cadre la presqu'île de Kola. Les phases accidentelle et surtout post-accidentelles, scénarisées par les Russes, devaient être gérées par les experts des deux instituts, russes et français; les autorités russes et la presse jouant leur propre rôle. 
La figure 5 schématise l'utilisation combinée d'ASTRAL et de PARIS lors de ces exercices. Il y figure notamment les échanges d'informations entre les utilisateurs des deux logiciels au cours du cycle ci dessous :

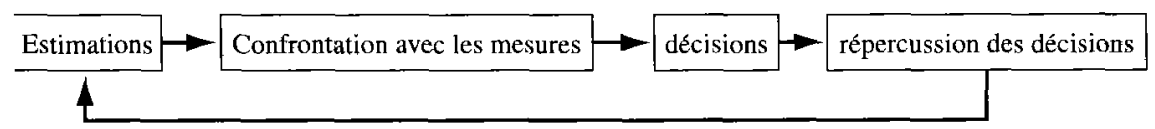

On note que les scénaristes mettent à la disposition des experts des bases de données de plus en plus élaborées et complètes, comme ce serait probablement le cas dans une situation réelle.
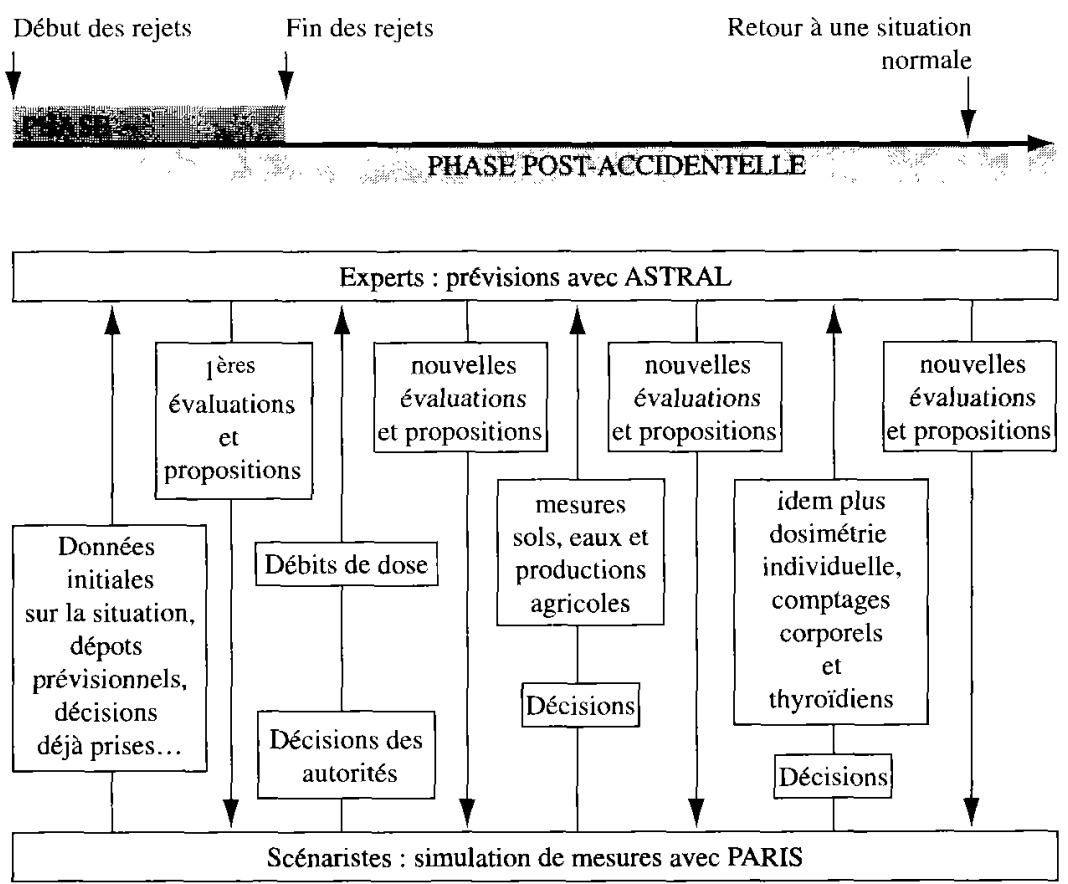

Fig. 5. - Schéma dynamique des interactions exercice de gestion post-accidentelle.

Dynamic diagram of an exercise of post-accidental management.

\section{Conclusion}

Le logiciel PARIS permet de simuler l'obtention de résultats de mesures en situation post-accidentelle. Sa structure fondée sur l'utilisation de bases de données est particulièrement bien adaptée à cette fonction. Il en est de même 
de l'interface utilisateur qui permet très rapidement de constituer les bases souhaitées.

PARIS propose une approche particulièrement réaliste d'une situation post-accidentelle et de sa gestion. Les bases de données générées sont d'une grande richesse qui rend compte de la diversité des informations que les experts auraient à traiter dans une telle situation. Il constitue ainsi la synthèse informatisée des enseignements opérationnels que l'on peut tirer de l'accident de Tchernobyl.

L'utilisation combinée de PARIS et du système ASTRAL lors d'exercices de gestion de situations post-accidentelles, présente un grand intérêt pour la préparation des experts, notamment en ce qui concerne l'exploitation de résultats de mesures et leur substitution progressive aux résultats de calculs.

Une nouvelle version de PARIS utilisable sur n'importe quel site, notamment français, pour lequel on disposerait des données générales correspondantes est actuellement à l'étude. Afin de mieux spécifier ces futurs développements, l'IBRAE a récemment collaboré à la scénarisation de l'exercice «Becquerel » de simulation d'un rejet accidentel du réacteur expérimental OSIRIS de Saclay. A ce titre une adaptation de PARIS aux caractéristiques de ce type de réacteur et aux données spécifiques de l'Ile de France a été réalisée sous le nom d' «ENVELOPPE ».

\section{RÉFÉRENCES}

Maubert H., Renaud Ph., Fache Ph., Métivier J. M. (1996) ASTRAL, a software for the estimation of accident release of radionucleides in the environment. IRPA 9. Vienna April 1996.

IPSN - IBRAE (1994) Exercice de prise de décision en situation post-accidentelle en cas de contamination radioactive de zones étendues, Rapport général de L'Institut de Sécurité Nucléaire de l'Académie des Sciences de RUSSIE et de L'Institut de Protection et de Sûreté Nucléaire Français. Avril 1994. 